\title{
Identification of Autophagy-Related Genes in the Potato Psyllid, Bactericera cockerelli and Their Expression Profile in Response to 'Candidatus Liberibacter Solanacearum' in the Gut
}

\author{
Xiao-Tian Tang and Cecilia Tamborindeguy * \\ Department of Entomology, Texas A\&M University, College Station, TX 77843, USA; xiaotian.tang@yale.edu \\ * Correspondence: ctamborindeguy@tamu.edu
}

check for

updates

Citation: Tang, X.-T.;

Tamborindeguy, C. Identification of

Autophagy-Related Genes in the

Potato Psyllid, Bactericera cockerelli

and Their Expression Profile in

Response to 'Candidatus Liberibacter

Solanacearum' in the Gut. Insects

2021, 12, 1073. https://doi.org/

$10.3390 /$ insects 12121073

Academic Editor: Nabil Killiny

Received: 21 October 2021

Accepted: 26 November 2021

Published: 30 November 2021

Publisher's Note: MDPI stays neutral with regard to jurisdictional claims in published maps and institutional affiliations.

Copyright: (c) 2021 by the authors. Licensee MDPI, Basel, Switzerland. This article is an open access article distributed under the terms and conditions of the Creative Commons Attribution (CC BY) license (https:// creativecommons.org/licenses/by/ $4.0 /)$.
Simple Summary: In North America, the bacterial plant pathogen 'Candidatus Liberibacter solanacearum' (Lso) infects solanaceous plants. Currently, Lso haplotypes, LsoA and LsoB are transmitted by potato psyllid, Bactericera cockerelli (Šulc). Because these bacteria are transmitted in a circulative and persistent manner, the gut of the psyllid is the first organ they encounter and could be a barrier to its transmission. Therefore, it is important to understand the molecular mechanisms involved in Lso acquisition and transmission. This study explored if an autophagic response was triggered in response to LsoA and/or LsoB in the gut of the adult potato psyllid. The results showed that Lso may induce the autophagic response in the adult psyllid gut since the majority of autophagy-related genes (ATGs) are sensitive and responsive to the exposure or infection of both LsoA and LsoB. Therefore, this study represents a stepping-stone towards understanding the molecular mechanisms involved in Lso transmission.

Abstract: Autophagy, also known as type II programmed cell death, is a cellular mechanism of "self-eating". Autophagy plays an important role against pathogen infection in numerous organisms. Recently, it has been demonstrated that autophagy can be activated and even manipulated by plant viruses to facilitate their transmission within insect vectors. However, little is known about the role of autophagy in the interactions of insect vectors with plant bacterial pathogens. 'Candidatus Liberibacter solanacearum' (Lso) is a phloem-limited Gram-negative bacterium that infects crops worldwide. Two Lso haplotypes, LsoA and LsoB, are transmitted by the potato psyllid, Bactericera cockerelli and cause damaging diseases in solanaceous plants (e.g., zebra chip in potatoes). Both LsoA and LsoB are transmitted by the potato psyllid in a persistent circulative manner: they colonize and replicate within psyllid tissues. Following acquisition, the gut is the first organ Lso encounters and could be a barrier for transmission. In this study, we annotated autophagy-related genes (ATGs) from the potato psyllid transcriptome and evaluated their expression in response to Lso infection at the gut interface. In total, 19 ATGs belonging to 17 different families were identified. The comprehensive expression profile analysis revealed that the majority of the ATGs were regulated in the psyllid gut following the exposure or infection to each Lso haplotype, LsoA and LsoB, suggesting a potential role of autophagy in response to Lso at the psyllid gut interface.

Keywords: autophagy; Candidatus Liberibacter solanacearum; Bactericera cockerelli; gut; gene expression

\section{Introduction}

Autophagy, which means "self-eating", is a conserved cellular degradation process that serves to deliver cytoplasmic proteins and organelles to lysosomes for degradation [1]. It plays important roles in many biological processes, such as maintaining homeostasis and preventing nutritional and metabolic-mediated stresses in eukaryotic organisms ranging from yeast to insects and mammals [2]. Autophagy is also an important component in the innate immune defense against infection by viral, bacterial, and fungal pathogens in animals [3]. 
In the last two decades, a series of autophagy-related genes (ATGs) have been characterized from lower (e.g., yeast) to higher eukaryotes (e.g., mammals and insects) [4-6]. Most but not all the ATGs play roles in the canonical autophagy pathway, regulating the formation of the autophagosome, a vesicular structure that is the hallmark of autophagy [7]. In yeast, more than 30 ATGs were identified, and around 20 of those are required for the efficient formation of sealed autophagosomes that proceed to fuse with lysosomes. In Drosophila, nearly all the core autophagy genes function in the autophagy pathway $[6,8,9]$. Under normal conditions, autophagy is kept at a low level, but it can be activated by starvation or other stress signals, which are known to induce the expression of some ATG genes [10]. Therefore, the identification of genes that participate in autophagy should establish a foundation for furthering our understanding of the molecular mechanism and the potential roles of autophagy in different physiological processes.

In insects, previous studies have revealed that autophagy components are important for Drosophila defense against viruses, such as vesicular stomatitis virus [11]. An increasing amount of evidence shows that autophagy could be induced also by plant pathogens in hemipteran insects, in particular by vector-borne plant viruses. The induced autophagy may play different roles: it could be a defensive mechanism to protect the insect against the invasion of the pathogens, or it could help the pathogen be acquired or escape specific tissues within the vector. For instance, Tomato yellow leaf curl virus (TYLCV) induces autophagy in its insect vector, the whitefly (Bemisia tabaci), and this induction results in decreased transmission while the inhibition of this response facilitates virus transmission [12]. In contrast, Chen, et al. [13] found that autophagy is induced by Rice gall dwarf virus (RGDV) in the intestine of its insect vector, the rice leafhopper (Recilia dorsalis). Further investigation demonstrated that the induction of autophagy improves the transmission of RGDV, whereas its inhibition blocks viral transmission. However, fewer studies have assessed the induction or inhibition of autophagy by vector-borne plant pathogenic bacteria and its potential role in bacterial transmission. This question needs to be assessed because autophagy is also induced in response to bacteria, for example, autophagy is involved regulating the population of the endosymbionts in Drosophila and beetles $[14,15]$.

Candidatus Liberibacter solanacearum' (Lso) is a phloem-limited, Gram-negative, unculturable bacterium [16]. It is a pathogen that infects members of the Apiaceae and Solanaceae plant families and causes great economic losses. Presently, several Lso haplotypes have been identified in the world [17-22]. Haplotypes LsoA and LsoB are transmitted by the potato psyllid (also known as tomato psyllid), Bactericera cockerelli, and infect numerous solanaceous plants (e.g., potatoes and tomatoes) in North America [23,24]. Lso has recently been found associated with other psyllids in the USA (e.g., psyllids associated with ash trees and Rhamnus) but whether they are transmitted to plants has not been established yet $[25,26]$. Both LsoA and LsoB are associated with potato zebra chip, a disease that has been responsible for losses of millions of dollars in potato producing regions [27]. Lso is transmitted by psyllids in a circulative and persistent manner [28-30]. Therefore, the psyllid gut is the first organ that Lso encounters. This organ could act as a barrier for Lso transmission and determine Lso transmission efficiency. Indeed, the ability of the bacteria to infect the gut depends on the insect immune responses as well as the bacterial strategies deployed to disrupt the host immunity.

In our previous studies, we evaluated whether Lso might induce apoptosis in psyllid guts. We did not find evidence of apoptosis in response to either LsoA or LsoB; further, we showed that Lso might repress the apoptotic response in the psyllid guts at an early stage of the infection for acquisition and transmission [31,32]. In this study, we aim to understand whether there might be an autophagic immune response in the psyllid gut following exposure to Lso. Toward this end, we first examined the potato psyllid transcriptome [33] to identify ATGs. Because there are few studies thoroughly investigating ATGs in hemipteran insects [34], we also annotated ATGs in representative Sternorrhyncha species including the pea aphid (Acyrthosiphon pisum), the whitefly (B. tabaci), and the Asian citrus psyllid (Diaphorina citri) which genomes have been sequenced [35-38]. Second, we evaluated the 
expression profiles of ATGs in response to LsoA or LsoB infection in the psyllid gut. We focused on the autophagic response of adult psyllids from infected colonies, which most likely acquired the bacteria during the nymphal stages, as well as in the responses that occur during the early infection. This study represents a first step towards understanding the molecular mechanisms and the potential role of autophagy in potato psyllid and Lso interactions.

\section{Materials and Methods}

\subsection{Insect Colonies and Tomato Plants}

Lso-free, LsoA- and LsoB-infected psyllid colonies were obtained as described in Yao, et al. [39]. These colonies were maintained separately on tomato plants (Moneymaker; Victory Seed Company, Molalla, OR, USA $)$ in insect-proof cages $(24 \mathrm{~cm} \times 13.5 \mathrm{~cm} \times 13.5 \mathrm{~cm}$, BioQuip ${ }^{\circledR}$, Compton, CA, USA) at room temperature $24 \pm 2{ }^{\circ} \mathrm{C}$ and under a photoperiod of $16: 8 \mathrm{~h}(\mathrm{~L}: \mathrm{D})$.

Tomato plants infected with Lso were obtained as described in Nachappa, et al. [40]. Briefly, six-week-old tomato plants were infected using three male psyllids harboring LsoA or LsoB, respectively. One week later, the psyllids were removed from the cages and the plants were tested for Lso infection using the LsoF/OI2 primers [41] three weeks after insect removal. Further, the Lso haplotype in the plants was confirmed using the Lso SSR-1 primers [19].

\subsection{Identification and Validation of Autophagy-Related Genes}

Genes potentially involved in autophagy in hemipteran insects were identified through blast searches of the potato psyllid transcriptome [33] and the genome of the pea aphid, the whitefly, and the Asian citrus psyllid [35-38] using the 20 D. melanogaster predicted ATGs as query (e-value cut off $1 \times 10^{-20}$ ) [42]. The D. melanogaster ATGs are from the FlyBase: https:// flybase.org/reports/FBgg0000076.html (accessed on 15 January 2019).

Specific primers for each candidate gene were designed (Table S1). RNA from a pool of 50 adult psyllids was purified using the RNeasy Mini Kit (Qiagen, Hilden, Germany), followed by a Dnase I treatment with Turbo Dnase (Ambion, Invitrogen, Waltham, MA, USA). Then, cDNA was produced using the Verso cDNA Synthesis kit (Thermo, Waltham, MA, USA) and anchored-Oligo (dT) primers according to the manufacturer's manual. Candidate genes were amplified by PCR. The PCR conditions were $95^{\circ} \mathrm{C}$ for $2 \mathrm{~min}$; followed by 35 cycles of $95^{\circ} \mathrm{C}$ for $30 \mathrm{~s}, 60^{\circ} \mathrm{C}$ for $30 \mathrm{~s}$, and $72{ }^{\circ} \mathrm{C}$ for $30-90 \mathrm{~s}$ (depending on the amplicon size); and a final extension at $72{ }^{\circ} \mathrm{C}$ for $5 \mathrm{~min}$. Amplicons were resolved in a $1 \%$ agarose gel and they were purified using the PureLink Quick Gel Extraction kit (Invitrogen, Waltham, MA, USA). Each PCR fragment (150 ng) was cloned into the pGEM-T easy vector using the pGEM-T easy cloning kit (Promega, Madison, WI, USA) and transformed into NovaBlue Singles Competent cells (Novagen, Temecula, CA, USA). For each candidate, the plasmid from at least three colonies was sequenced by Eton Bioscience Inc. (San Diego, CA, USA). The obtained sequences were compared to the bioinformatics predictions.

\subsection{Expression of Autophagy-Related Genes}

The expression of candidate ATGs was evaluated in the psyllid gut upon Lso infection. To obtain newly infected insects, approximately one-week-old female adult psyllids from the Lso-free colony were transferred to LsoA- or LsoB-infected plants. The insects were collected 2, 3, 5, and 7 days later. Insects from the Lso-free colony were used as a control. Three replicates were analyzed for each treatment, and each replicate had 200 psyllid individuals. After exposure, the psyllid guts were dissected in $1 \times$ PBS with RNAlater (Ambion, Invitrogen, Waltham, MA, USA) under the stereomicroscope (Olympus, Tokyo, Japan) as described in Ibanez, et al. [43]. The guts of female adult psyllids from the Lso-free, LsoA- and LsoB-infected colonies were dissected as described above. RNA purification and cDNA synthesis from each sample was performed as described above. The expression of ATGs was evaluated by quantitative real-time PCR (qPCR) using the SensiFAST SYBR 
Hi-ROX kit (Bioline, London, UK) following the manufacturer's manual. For each reaction, $25 \mathrm{ng}$ of cDNA, $250 \mathrm{nM}$ of each primer (Table S1), and $1 \times$ of SYBR Green Master Mix were mixed. The volume was adjusted to $10 \mu \mathrm{L}$ with nuclease-free water. The qPCR conditions were as follows: $95^{\circ} \mathrm{C}$ for $2 \mathrm{~min}$ followed by 40 cycles at $95^{\circ} \mathrm{C}$ for $5 \mathrm{~s}$ and $60{ }^{\circ} \mathrm{C}$ for $30 \mathrm{~s}$. The qPCR assays were performed using a QuantStudio ${ }^{\mathrm{TM}} 6$ Flex Real-Time PCR System (Applied Biosystems, Foster City, CA, USA). All reactions were performed in triplicates and negative controls were included in each run. The relative expression of the candidate genes was estimated with the delta-delta CT method [44] using elongation factor-1a (GenBank KT185020) and ribosomal protein subunit 18 (GenBank KT279693) [45] as reference genes.

\subsection{Data Analysis}

All statistical analyses were conducted with JMP Version 12 (SAS Institute Inc., Cary, NC, USA). Regulation of ATGs was determined using one-way ANOVA with Tukey's post hoc test.

\section{Results}

\subsection{Autophagy-Related Genes in Potato Psyllid and Other Hemipteran Insects}

A total of 19 ATG homologs were identified and their sequences were validated in the potato psyllid via transcriptome search. These genes were classified into 17 ATG gene families. The gene names and abbreviations used hereafter are listed in Table 1. In parallel, 22,19 , and 27 ATG were also identified in the genome of the pea aphid, the whitefly, and the Asian citrus psyllid, respectively (Table S2). With the exception of ATG4a, at least one putative homolog to the Drosophila ATGs was identified in the B. cockerelli transcriptome; those included serine/threonine protein kinase ULK2 (BcATG1), BcATG2-BcATG5, Beclin1 (BcATG6), BcATG7, gamma-aminobutyric acid receptor-associated protein (BcATG8), $B c A T G 9, B c A T G 10, B c A T G 12, B c A T G 13$, beclin 1-associated autophagy-related key regulator (BcATG14), BcATG16, RB1-inducible coiled-coil protein 1-like (BcATG17), WD repeat domain phosphoinositide-interacting proteins (BcATG18), and BcATG101. Further, ATGs from each Drosophila ATG family were identified in the pea aphid and the whitefly genomes, however, ATG12 and ATG14 were absent in the current Asian citrus psyllid genome.

Table 1. Name of B. cockerelli autophagy-related genes and their codes used in this paper.

\begin{tabular}{|c|c|}
\hline Gene Name & Code \\
\hline Serine/threonine-protein kinase ULK2-like isoform 1 & BcATG1 \\
\hline Autophagy-related protein 2-like & BcATG2 \\
\hline Autophagy related protein Atg3-like protein & BcATG3 \\
\hline Cysteine protease ATG4B-like isoform 1 & BcATG4B \\
\hline Autophagy protein 5 & BcATG5 \\
\hline Beclin-1-like protein & BcATG6 \\
\hline Ubiquitin-like modifier-activating enzyme ATG7 & BcATG7 \\
\hline Gamma-aminobutyric acid receptor-associated protein & BcATG8 \\
\hline Autophagy-related protein 9A & BcATG9 \\
\hline Ubiquitin-like-conjugating enzyme ATG10 & BcATG10 \\
\hline Autophagy protein 12-like & BcATG12 \\
\hline Autophagy-related protein 13 homolog & BcATG13 \\
\hline Beclin 1-associated autophagy-related key regulator-like & BcATG14 \\
\hline Autophagy-related protein $16-1$-like & BcATG16 \\
\hline RB1-inducible coiled-coil protein 1-like & BcATG17 \\
\hline WD repeat domain phosphoinositide-interacting protein 2 & BcATG18-2 \\
\hline WD repeat domain phosphoinositide-interacting protein 3 & BcATG18-3 \\
\hline WD repeat domain phosphoinositide-interacting protein 4 & BcATG18-4 \\
\hline Autophagy-related protein 101-like isoform 1 & BcATG101 \\
\hline
\end{tabular}




\subsection{Expression of Autophagy-Related Genes upon Lso Infection}

The expression pattern of the identified potato psyllid ATGs was evaluated in the gut of adults following specific acquisition access periods on Lso-infected plants. Differences in the expression of ATGs were identified upon LsoA and LsoB acquisition.

Upon LsoA acquisition, 15 of the 19 ATGs were regulated. Among those, BcATG2 was only up-regulated at day 2 compared to all other time points or compared to the uninfected guts, while $B C A T G 6$ and $B c A T G 16$ were up-regulated at day 2 compared to the uninfected guts. BcATG1, BcATG101, BcATG17, BcATG9, BcATG14, BcATG5, BcATG12, $B c A T G 3$, and BcATG7 remained up-regulated longer. BcATG13, BcATG18-2, BcATG12, $B c A T G 3, B C A T G 7$, and $B c A T G 8$ were expressed at higher levels at days 3 or 5 . Four genes (BcATG18-3, BcATG18-4, BcATG4B, and BcATG10) were not significantly regulated upon LsoA infection (Figure S1). Overall, a majority of genes potentially involved in the steps previous to the phagophore formation were up-regulated upon LsoA acquisition (Figure 1).
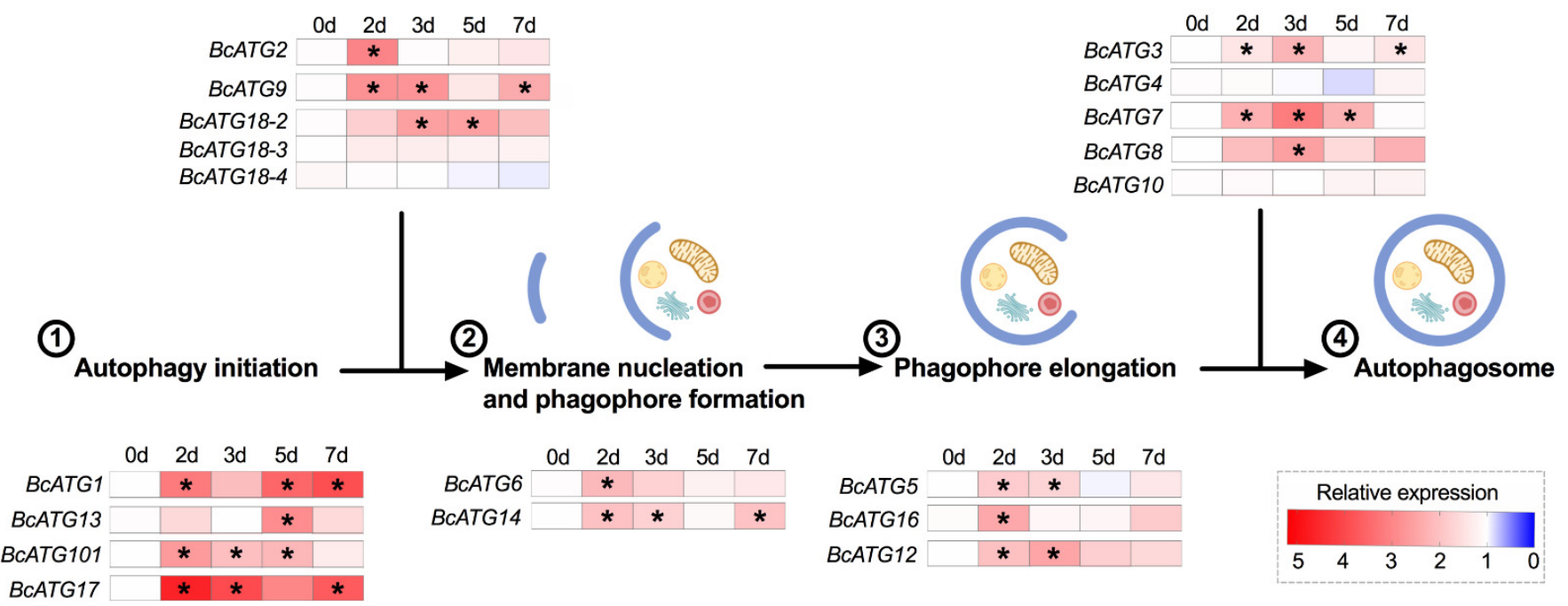

Figure 1. Heat map of gene expression profiles of ATGs upon LsoA infection corresponding to the process of autophagosome formation. $0 \mathrm{~d}$ indicates the control, in which psyllids were taken from the Lso-free colony; $2 \mathrm{~d}, 3 \mathrm{~d}, 5 \mathrm{~d}$, and $7 \mathrm{~d}$ indicate the psyllids were infected by LsoA for 2, 3, 5, and 7 days, respectively ( $\mathrm{d}$ indicates day). The numbers indicate the steps of autophagosome formation. The asterisks $\left(^{*}\right)$ indicate statistical differences compared to control at $p<0.05$ using one-way ANOVA with Tukey's post hoc test.

Upon LsoB acquisition, 10 of the 19 ATGs were regulated and multiple members had similar expression patterns. First, $B C A T G 18-3$ was only up-regulated at day 2 compared to the uninfected guts or day 7, while BcATG1 and BcATG18-2 were also up-regulated at day 2 but remained up-regulated longer and/or at later time points. BCATG101, BCATG17, $B c A T G 14, B c A T G 12, B c A T G 7$, and BCATG8 were expressed at higher levels at days 3 or 5 . Nine genes were not significantly regulated upon LsoB infection (Figure 2 and Figure S2). 


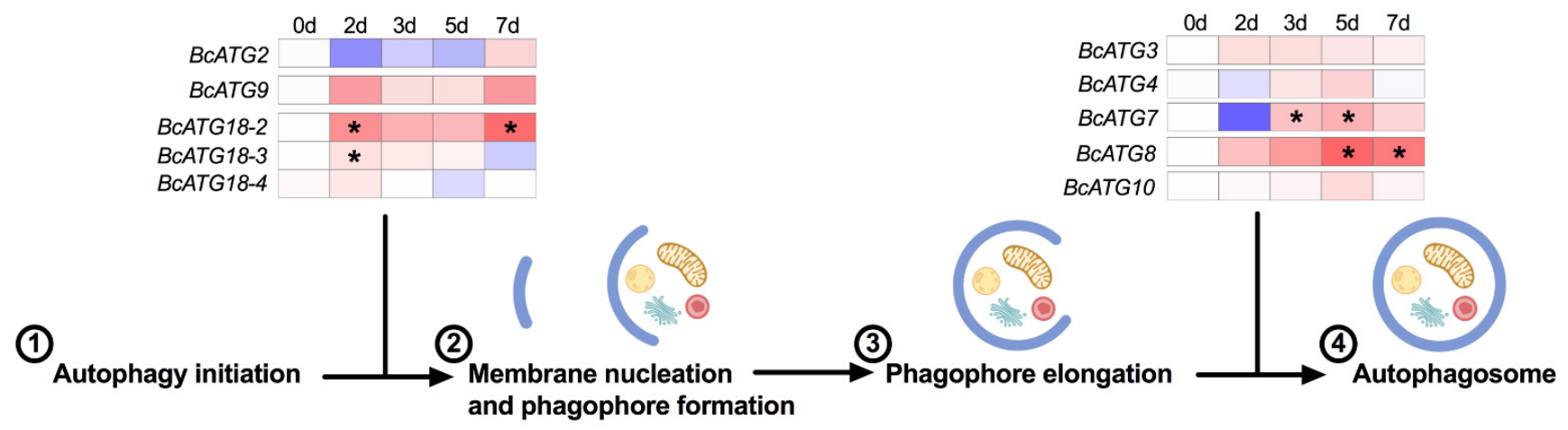

\begin{tabular}{r|c|c|c|c|c|} 
& Od & $2 \mathrm{~d}$ & $3 \mathrm{~d}$ & $5 \mathrm{~d}$ & $7 \mathrm{~d}$ \\
\hline BCATG1 & & $*$ & $*$ & $*$ & $*$ \\
\hline BCATG13 & & & & & \\
\hline BCATG101 & & & $*$ & & \\
\hline BCATG17 & & & $*$ & $*$ & $*$
\end{tabular}
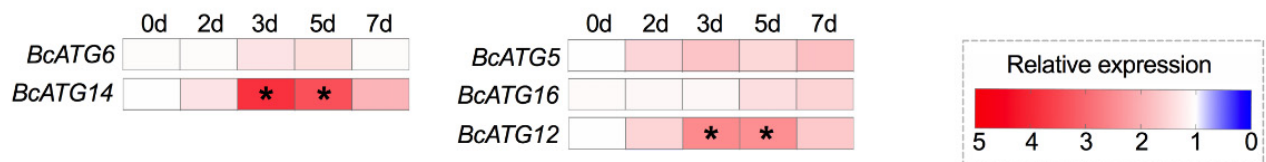

Figure 2. Heat map of gene expression profiles of ATGs upon LsoB infection corresponding to the process of autophagosome formation. $0 \mathrm{~d}$ indicates the control, in which psyllids were taken from the Lso-free colony; $2 \mathrm{~d}, 3 \mathrm{~d}, 5 \mathrm{~d}$, and $7 \mathrm{~d}$ indicate the psyllids were infected by LsoB for 2, 3, 5, and 7 days, respectively ( $d$ indicates day). The numbers indicate the steps of autophagosome formation. The asterisks $\left(^{*}\right)$ indicate statistical differences compared to control at $p<0.05$ using one-way ANOVA with Tukey's post hoc test.

\subsection{Expression of Autophagy-Related Genes in Response to Persistent Lso Infection}

The expression pattern of the identified ATGs was evaluated in the gut of adults from the Lso-infected psyllid colonies, which most likely acquired the bacteria during the nymphal stages. We found that approximately half of the ATGs were significantly regulated in response to LsoA or LsoB infection. Eight ATG genes (BcATG1, BcATG9, BcATG18-2, $B c A T G 6, B c A T G 16, B c A T G 7, B c A T G 8$, and $B c A T G 10)$ were significantly up-regulated in response to LsoA, while $B c A T G 17$ was down-regulated. However, five genes (BcATG1, $B c A T G 17, B c A T G 18-2, B c A T G 14$, and $B c A T G 8)$ were significantly up-regulated in response to LsoB. Taken together, three genes (BcATG1, BcATG18-2, and BcATG8) were significantly up-regulated in response to both LsoA and LsoB (Figure S3). Overall, genes across all the stages of autophagosome formation were significantly regulated (Figure 3).

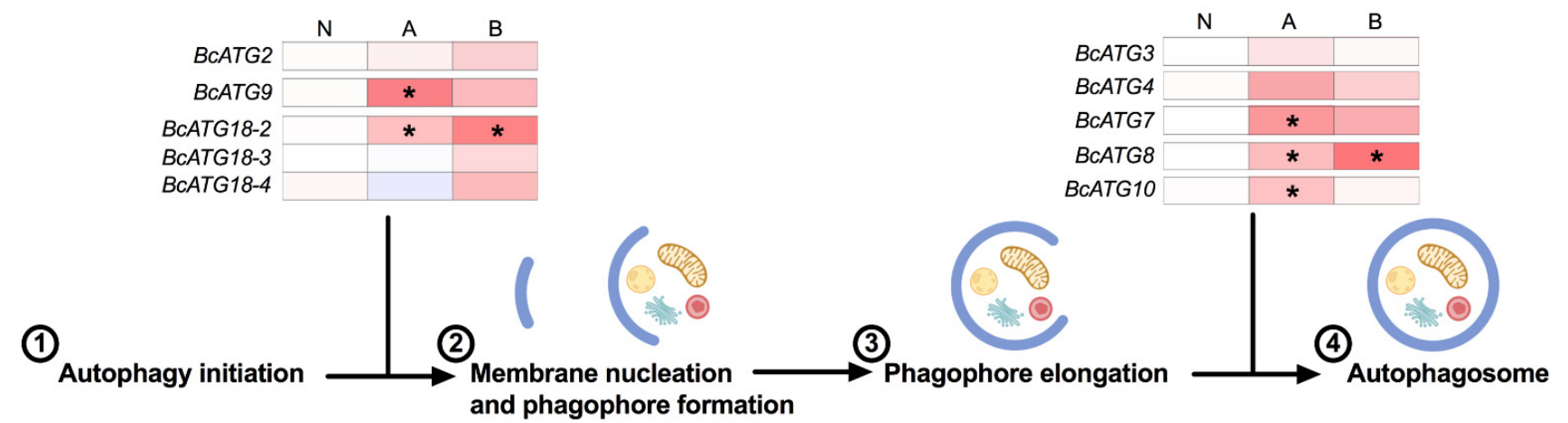

\begin{tabular}{r|c|c|c} 
& N & A & B \\
\cline { 2 - 3 } BCATG1 & & $*$ & $*$ \\
\cline { 2 - 4 } BCATG13 & & & \\
BCATG101 & & & \\
BCATG17 & & $*$ & $*$ \\
\cline { 2 - 4 } & & &
\end{tabular}

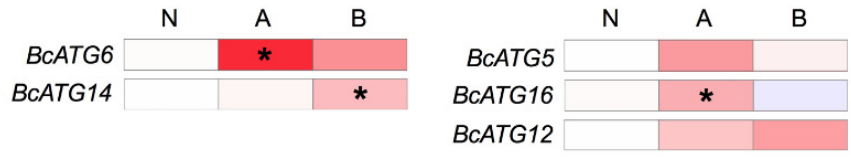

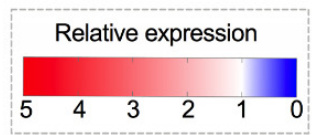

Figure 3. Heat map of gene expression profiles of ATGs in response to LsoA and LsoB persistent infection corresponding to the process of autophagosome formation. N: Lso-free colonies; A and B indicate the psyllids were infected by LsoA and LsoB, respectively. The numbers indicate the steps of autophagosome formation. The asterisks $\left(^{*}\right)$ indicate statistical differences compared to control at $p<0.05$ using one-way ANOVA with Tukey's post hoc test. 


\section{Discussion}

Autophagy is a self-regulated process and is highly conserved from yeast to mammals [10]. The role of autophagy is not limited to the conditions under the stimulus of starvation. It also has a significant role in innate immunity. Indeed, autophagy is a cellular degradation process that can capture and eliminate intracellular microbes by delivering them to lysosomes for destruction. In addition to directly degrading pathogens, autophagy can also activate the host immune system [46]. In mammals, autophagy provides an excellent intracellular defense system against intracellular pathogens, such as Listeria monocytogenes and Shigella flexneri $[47,48]$. Take S. flexneri for example, after bacterial uptake into host cells, the bacterial peptidoglycans can be detected by nucleotide-binding oligomerization domain (NOD)-like receptors (NLRs), which can further interact with Atg16L1 and recruit other autophagy proteins to initiate autophagosome biogenesis in response to bacterial invasion [49]. In Drosophila, autophagy is also an essential component of immunity. For instance, Shelly, Lukinova, Bambina, Berman and Cherry [11] found that autophagy in Drosophila played a direct antiviral role against the vesicular stomatitis virus (VSV). Indeed, silencing in D. melanogaster S2 cells of several autophagy-related genes including ATG1, ATG5, ATG8a/LC3, and ATG18/WIPI2 followed by viral challenge resulted in increased VSV infection rate. Similarly, upon VSV infection, mutant flies depleted for ATG18/WIPI2 presented increased viral replication compared to control flies as well as increased mortality when compared to unchallenged mutant flies. In addition to virus response, the studies also indicated that autophagy components are important for $D$. melanogaster defense against infection with bacteria, such as Escherichia coli and Wolbachia [14,50]. For example, RNAi knockdown of autophagy components (e.g., ATG5, ATG7, and ATG12) caused an increased pathogen load and decreased survival upon E. coli infection [50].

The core machinery of autophagy can be classified into several functional gene units including the ATG1/ULK1 (Uncoordinated-51 like kinase) complex (ULK1, ULK2, ATG13, ATG17, ATG101, FIP200, etc.), the transmembrane protein ATG9 cycling system (ATG2, ATG9, and ATG18), the class III phosphoinositide 3-kinase [PI(3)K] complex (ATG14L, VPS34, Beclin-1, and VPS15), and two ubiquitin-like conjugating systems, ATG12-ATG5 and ATG8/LC3 (light chain 3) [51]. The canonical autophagosome formation process can be subdivided into several phases, including autophagy initiation, membrane nucleation, phagophore formation, phagophore elongation, and autophagosome completion [52,53]. First, the ULK/ATG1 and ATG9 complex recruit the autophagy-specific PI(3)K to generate phosphatidylinositol 3-phosphate $[\mathrm{PI}(3) \mathrm{P}]$ required for membrane nucleation and phagophore formation. To start elongation, the phagophore recruits PI(3)P-binding complex (ATG18/WIPI and ATG2) and then binds ATG5-ATG12/ATG16L1 complex. Finally, the accumulation of those proteins initiates the conjugation of microtubule-associated protein LC3/ATG8 family members to the phagophore and forms the autophagosome $[9,53]$.

Although the ATG genes are very critical for autophagosome formation, it is now clear that ATG genes also have autophagy-independent roles in immunity [54]. A recent study in mammalian cells found that ATG8/LC3 can recruit innate immune proteins (e.g., GTPases) for a type of antiviral defense that appears to not involve fusion with the lysosome [55]. Other core machinery autophagy components, such as ULK1, FIP200, ATG14L, ATG16L1 and ATG9, are also recruited to the bacteria Salmonella, and each of them has a role in restricting the intracellular growth of Salmonella in humans [56]. These new insights would likely stimulate further analysis of autophagy and autophagy proteins in other organisms, including Drosophila and other insects. In particular, these studies could determine whether these proteins act in canonical autophagy, noncanonical autophagy, or some other process to provide an immune defense.

In this study, we annotated autophagy-related genes in the potato psyllid and we evaluated their expression in the gut indeed following Lso exposure or infection. A significant change in expression was measured in response to Lso. These genes were regulated in a temporal manner showing different regulation profiles. Upon LsoA infection, most of the ATGs were significantly up-regulated at a 2-day infection, probably at a time 
when Lso first began to translocate into the gut cells. In particular, the ATGs involved in autophagy initiation and phagophore formation showed a more intense up-regulation profile. For instance, $B C A T G 1$ and its subunit BCATG17, which control the initiation of autophagosome formation, both expressed extensive up-regulation profiles. The induction of six ATGs was delayed until day 3 or day 5 upon exposure to LsoA. Furthermore, nine genes remained induced for a longer period of time. Finally, five genes (BcATG1, BcATG17, $B c A T G 9, B c A T G 14$, and BcATG3) were still up-regulated at day 7. However, the expression of the other tested genes had been reduced to their prior levels. The transcriptional regulation of ATG genes is critical for autophagy. Indeed, the majority of the ATG genes are up-regulated upon stress [57].

The regulation of ATGs upon LsoB infection showed a different pattern compared to LsoA, and approximately half of those genes were regulated during the 7 days analyzed. Specifically, only three genes (BCATG1, BcATG18-2, and BcATG18-3), which contribute to the autophagy initiation and phagophore formation, were significantly up-regulated at the beginning of acquisition ( 2 days). The other seven genes were induced after 3 days of infection. Overall, the induction of ATGs upon LsoB infection was delayed and/or at a lower level when compared to their expression upon LsoA infection. For instance, BcATG8, a ubiquitin-like protein required for the formation of autophagosomal membranes, was significantly up-regulated at day 3 in response to LsoA, while it was induced after day 5 upon LsoB infection. This delayed response probably allows LsoB to be able to replicate and translocate through the gut epithelium faster. Indeed, in a previous study, we have shown that LsoB accumulates faster and to higher levels in the gut of adult psyllids. Further, this difference in accumulation correlates with reduced latency and higher transmission efficiency of LsoB than LsoA [31]. We also noted that several genes (e.g., BcATG13, BcATG101, and $B c A T G 2)$ that function before phagophore formation showed a down-regulated profile (but not significant) upon LsoB infection, probably indicating an early repression of the autophagic response by LsoB in the psyllid gut to some extent. Indeed, although bacteria can be targeted and eliminated by autophagy, some of them have evolved strategies to escape this defense system or even hijack the autophagy machinery to promote their intracellular growth [54]. Importantly, some bacteria inhibit autophagy by directly interfering with the activity of autophagy components. For instance, Legionella pneumophila avoid autophagy using its effector protein by mimicking the function of ATG4B-like cysteine protease of the host that directly targets the amide bond between the tyrosine and the glycine at the $C$ terminus of LC3/ATG8, which is covalently linked to phosphatidylethanolamine (PE) during the autophagy induction to form LC3-PE; therefore, further inhibiting autophagosome formation [58]. We hypothesize that LsoB can repress (or not induce to the same level) the autophagic response in the psyllid gut at the early stages of infection while LsoA could not. Indeed, the early LsoA infection significantly up-regulated all the ATGs involved in autophagy induction in other hemipteran insects by plant viruses. Specifically, three autophagy-related genes (Ulk1/Atg1, Atg5, and Atg8) increased rapidly and significantly after $48 \mathrm{~h}$ post inoculation of RGDV in the rice leafhopper [13]; furthermore, the expression of the three ATG genes (Atg3, Atg9 and Atg12) increased significantly in TYLCV-infected whiteflies [12]. In our study, BcATG1, BcATG5, BcATG3, BcATG9, and BcATG12 were significantly regulated after 2 days of LsoA infection, and $B C A T G 8$ was expressed at higher levels at day 3. However, only three of those genes (BcATG1, BcATG12, and BcATG8) were induced upon LsoB infection. Therefore, we hypothesized that autophagy could be induced in the gut of the adult psyllid upon LsoA infection, and this induction may be linked to the reduced increase of LsoA titer in the gut of psyllids compared to LsoB and its delayed transmission. It is unclear whether autophagy is induced in response to LsoB. If induced, this response is probably delayed compared to the induction by LsoA.

In addition, from the ATGs expression profile in response to persistent Lso infection, we also found that several ATGs were up-regulated in response to LsoA or LsoB and that more ATGs were up-regulated in response to LsoA. This observation is consistent with the ATGs induction upon Lso infection. Importantly, differences in virulence between these two 
haplotypes were determined in association with their host plants and insect vector: in both cases, LsoB was found to be more pathogenic $[39,59,60]$. It is, therefore, possible that LsoB is able to better defend itself against the psyllid immunity, or even also manipulate those defenses to its advantage. We also noted that BcATG17 was significantly down-regulated in response to persistent LsoA infection, indicating a putative repression of the autophagic response. However, both LsoA and LsoB significantly induced the core autophagy protein, $B c A T G 8$. Therefore, we hypothesized that autophagy could be occurring in the gut of the adult psyllid in response to persistent LsoA and LsoB infection. However, the autophagic immune response cannot be an efficient defense because both LsoA and LsoB can be successfully transmitted by potato psyllids. The plausible explanation is that even if autophagy is induced in response to the pathogen, this defense strategy is not sufficient or not widespread enough to stop Lso.

\section{Conclusions}

In summary, we identified 19 homologs of autophagy-related genes from potato psyllid. Our study suggested that the expression of the majority of autophagy-related genes changed significantly after LsoA or LsoB exposure or in the case of established infection of the gut. Importantly, in the case of autophagy, the gene expression correlates with the actual autophagy [10]. Further investigations need to be conducted to deepen the study of the autophagic response of potato psyllid to Lso infection and its role in Lso transmission. A combination of methods, such as western blotting, immunostaining and transmission electron microscopy, as well as RNAi-mediated gene silencing to analyze the autophagic response are recommended.

Supplementary Materials: The following are available online at https:/ /www.mdpi.com/article/ 10.3390/insects12121073/s1, Figure S1: Regulation of ATGs in the psyllid gut upon LsoA infection. Data represent means \pm SD of three independent experiments. Different letters indicate statistical differences at $p<0.05$ using one-way ANOVA with Tukey's post hoc test. N: Lso-free colonies; $2 \mathrm{~d}$, $3 \mathrm{~d}, 5 \mathrm{~d}$, and $7 \mathrm{~d}$ indicate the psyllids were infected by LsoA for 2, 3, 5, and 7 days, respectively. The numbers indicate the steps of autophagosome formation. Figure S2: Regulation of ATGs in the psyllid gut upon LsoB infection. Data represent means $\pm S D$ of three independent experiments. Different letters indicate statistical differences at $p<0.05$ using one-way ANOVA with Tukey's post hoc test. N: Lso-free colonies; $2 \mathrm{~d}, 3 \mathrm{~d}, 5 \mathrm{~d}$, and $7 \mathrm{~d}$ indicate the psyllids were infected by LsoB for 2,3,5, and 7 days, respectively. The numbers indicate the steps of autophagosome formation. Figure S3: Regulation of ATGs in the psyllid gut in response to LsoA and LsoB persistent infection. Data represent means $\pm \mathrm{SD}$ of three independent experiments. Different letters indicate statistical differences at $p<0.05$ using one-way ANOVA with Tukey's post hoc test. N: Lso-free colonies; A and $B$ indicate the psyllids were infected by LsoA and LsoB, respectively. The numbers indicate the steps of autophagosome formation. Table S1: Primers for bioinformatics validation and gene expression analyses by qPCR of autophagy-related genes. Table S2: Comparison of autophagy-related genes in D. melanogaster, A. pisum, B. tabaci, D. citri and B. cockerelli.

Author Contributions: Conceptualization, X.-T.T. and C.T.; methodology, X.-T.T. and C.T.; software, X.-T.T. and C.T.; validation, X.-T.T. and C.T.; formal analysis, X.-T.T. and C.T.; investigation, X.-T.T. and C.T.; resources, C.T.; data curation, X.-T.T. and C.T.; writing-original draft preparation, X.-T.T. and C.T.; writing — review and editing, X.-T.T. and C.T.; visualization, X.-T.T. and C.T.; supervision, C.T.; project administration, C.T.; funding acquisition, C.T. All authors have read and agreed to the published version of the manuscript.

Funding: This research was funded by Texas A\&M University and Texas A\&M AgriLife Research (Controlling Exotic and Invasive Insect-Transmitted Pathogens) and Hatch project TEX0-1-9381. XT Tang received the Herb Dean'40 Endowed Scholarship from the Department of Entomology at Texas A\&M University.

Institutional Review Board Statement: Not applicable.

Informed Consent Statement: Not applicable.

Data Availability Statement: Not applicable. 
Acknowledgments: We would like to thank Maria Azucena Mendoza Herrera for her help maintaining insect and plant colonies. The authors thank the Agriculture Women Excited to Share Opinions, Mentoring and Experiences (AWESOME) faculty group of the College of Agriculture and Life Sciences at Texas A\&M University for assistance with editing the manuscript.

Conflicts of Interest: The authors declare no conflict of interest.

\section{References}

1. Mizushima, N.; Levine, B.; Cuervo, A.M.; Klionsky, D.J. Autophagy fights disease through cellular self-digestion. Nature 2008, 451, 1069. [CrossRef]

2. Khandia, R.; Dadar, M.; Munjal, A.; Dhama, K.; Karthik, K.; Tiwari, R.; Yatoo, M.; Iqbal, H.; Singh, K.P.; Joshi, S.K. A Comprehensive Review of Autophagy and Its Various Roles in Infectious, Non-Infectious, and Lifestyle Diseases: Current Knowledge and Prospects for Disease Prevention, Novel Drug Design, and Therapy. Cells 2019, 8, 674. [CrossRef]

3. Yano, T.; Kurata, S. Intracellular recognition of pathogens and autophagy as an innate immune host defence. J. Biochem. 2011, 150, 143-149. [CrossRef] [PubMed]

4. Suzuki, K.; Akioka, M.; Kondo-Kakuta, C.; Yamamoto, H.; Ohsumi, Y. Fine mapping of autophagy-related proteins during autophagosome formation in Saccharomyces cerevisiae. J. Cell Sci. 2013, 126, 2534-2544. [CrossRef]

5. Yang, Z.; Klionsky, D.J. Mammalian autophagy: Core molecular machinery and signaling regulation. Curr. Opin. Cell Biol. 2010, 22, 124-131. [CrossRef] [PubMed]

6. Zirin, J.; Perrimon, N. Drosophila as a Model System to Study Autophagy. Semin. Immunopathol. 2010, 32, 363-372. [CrossRef] [PubMed]

7. Levine, B.; Kroemer, G. Biological functions of autophagy genes: A disease perspective. Cell 2019, 176, 11-42. [CrossRef]

8. Nakatogawa, H.; Suzuki, K.; Kamada, Y.; Ohsumi, Y. Dynamics and diversity in autophagy mechanisms: Lessons from yeast. Nat. Rev. Mol. Cell Biol. 2009, 10, 458. [CrossRef]

9. Mizushima, N.; Yoshimori, T.; Ohsumi, Y. The role of Atg proteins in autophagosome formation. Annu. Rev. Cell Dev. Biol. 2011, 27, 107-132. [CrossRef]

10. He, C.; Klionsky, D.J. Regulation mechanisms and signaling pathways of autophagy. Annu. Rev. Genet. 2009, 43, 67-93. [CrossRef] [PubMed]

11. Shelly, S.; Lukinova, N.; Bambina, S.; Berman, A.; Cherry, S. Autophagy is an essential component of Drosophila immunity against vesicular stomatitis virus. Immunity 2009, 30, 588-598. [CrossRef]

12. Wang, L.-L.; Wang, X.-R.; Wei, X.-M.; Huang, H.; Wu, J.-X.; Chen, X.-X.; Liu, S.-S.; Wang, X.-W. The autophagy pathway participates in resistance to tomato yellow leaf curl virus infection in whiteflies. Autophagy 2016, 12, 1560-1574. [CrossRef] [PubMed]

13. Chen, Y.; Chen, Q.; Li, M.; Mao, Q.; Chen, H.; Wu, W.; Jia, D.; Wei, T. Autophagy pathway induced by a plant virus facilitates viral spread and transmission by its insect vector. PLoS Pathog. 2017, 13, e1006727. [CrossRef] [PubMed]

14. Voronin, D.; Cook, D.A.; Steven, A.; Taylor, M.J. Autophagy regulates Wolbachia populations across diverse symbiotic associations. Proc. Natl. Acad. Sci. USA 2012, 109, E1638-E1646. [CrossRef] [PubMed]

15. Vigneron, A.; Masson, F.; Vallier, A.; Balmand, S.; Rey, M.; Vincent-Monégat, C.; Aksoy, E.; Aubailly-Giraud, E.; Zaidman-Rémy, A.; Heddi, A. Insects recycle endosymbionts when the benefit is over. Curr. Biol. 2014, 24, 2267-2273. [CrossRef]

16. Hansen, A.; Trumble, J.; Stouthamer, R.; Paine, T. New Huanglongbing (HLB) Candidatus species," Ca. Liberibacter psyllarous" found to infect tomato and potato is vectored by the psyllid Bactericera cockerelli. Appl. Environ. Microbiol. 2008, 73, 7531-7535. [CrossRef] [PubMed]

17. Glynn, J.; Islam, M.; Bai, Y.; Lan, S.; Wen, A.; Gudmestad, N.; Civerolo, E.; Lin, H. Multilocus sequence typing of 'Candidatus Liberibacter solanacearum'isolates from North America and New Zealand. J. Plant. Pathol. 2012, 94, $223-228$.

18. Haapalainen, M.L.; Wang, J.; Latvala, S.; Lehtonen, M.T.; Pirhonen, M.; Nissinen, A.I. Genetic variation of 'Candidatus Liberibacter solanacearum'haplotype $\mathrm{C}$ and identification of a novel haplotype from Trioza urticae and stinging nettle. Phytopathology 2018, 108, 925-934. [CrossRef]

19. Lin, H.; Islam, M.S.; Bai, Y.; Wen, A.; Lan, S.; Gudmestad, N.C.; Civerolo, E.L. Genetic diversity of 'Cadidatus Liberibacter solanacearum'strains in the United States and Mexico revealed by simple sequence repeat markers. Eur. J. Plant. Pathol. 2012, 132, 297-308. [CrossRef]

20. Nelson, W.R.; Sengoda, V.G.; Alfaro-Fernandez, A.O.; Font, M.I.; Crosslin, J.M.; Munyaneza, J.E. A new haplotype of “Candidatus Liberibacter solanacearum" identified in the Mediterranean region. Eur. J. Plant. Pathol. 2013, 135, 633-639. [CrossRef]

21. Swisher Grimm, K.D.; Garczynski, S.F. Identification of a new haplotype of 'Candidatus Liberibacter solanacearum'in Solanum tuberosum. Plant. Dis. 2018, 103, 468-474. [CrossRef]

22. Mauck, K.E.; Sun, P.; Meduri, V.R.; Hansen, A.K. New Ca. Liberibacter psyllaurous haplotype resurrected from a 49-year-old specimen of Solanum umbelliferum: A native host of the psyllid vector. Sci. Rep. 2019, 9, 9530. [CrossRef] [PubMed]

23. Liefting, L.W.; Sutherland, P.W.; Ward, L.I.; Paice, K.L.; Weir, B.S.; Clover, G.R. A new 'Candidatus Liberibacter'species associated with diseases of solanaceous crops. Plant. Dis. 2009, 93, 208-214. [CrossRef] [PubMed]

24. Tamborindeguy, C.; Huot, O.B.; Ibanez, F.; Levy, J. The influence of bacteria on multi-trophic interactions among plants, psyllids, and pathogen. Insect Sci. 2017, 24, 961-974. [CrossRef] 
25. Wamonje, F.; Zhou, N.; Bamrah, R.; Wist, T.; Prager, S. Detection and identification of a 'Candidatus Liberibacter solanacerum'species from ash tree infesting psyllids. Phytopathology 2021, in press. [CrossRef] [PubMed]

26. Kwak, Y.; Sun, P.; Meduri, V.R.; Percy, D.M.; Mauck, K.E.; Hansen, A.K. Uncovering Symbionts Across the Psyllid Tree of Life and the Discovery of a New Liberibacter Species, “Candidatus” Liberibacter capsica. Front. Microbiol. 2021, 12, 739763. [CrossRef]

27. Munyaneza, J.E. Zebra chip disease of potato: Biology, epidemiology, and management. Am. J. Potato Res. 2012, 89, 329-350. [CrossRef]

28. Cicero, J.; Fisher, T.; Brown, J.K. Localization of 'Candidatus Liberibacter solanacearum'and Evidence for Surface Appendages in the Potato Psyllid Vector. Phytopathology 2016, 106, 142-154. [CrossRef] [PubMed]

29. Cicero, J.M.; Fisher, T.W.; Qureshi, J.A.; Stansly, P.A.; Brown, J.K. Colonization and Intrusive Invasion of Potato Psyllid by 'Candidatus Liberibacter solanacearum'. Phytopathology 2017, 107, 36-49. [CrossRef]

30. Cooper, W.R.; Sengoda, V.G.; Munyaneza, J.E. Localization of 'Candidatus Liberibacter solanacearum'(Rhizobiales: Rhizobiaceae) in Bactericera cockerelli (Hemiptera: Triozidae). Ann. Entomol. Soc. Am. 2014, 107, 204-210. [CrossRef]

31. Tang, X.-T.; Fortuna, K.; Mendoza Herrera, A.; Tamborindeguy, C. Liberibacter, a preemptive bacterium: Apoptotic response repression in the host gut at the early infection to facilitate its acquisition and transmission. Front. Microbiol. 2020, 11, 3348. [CrossRef]

32. Tang, X.-T.; Jing, X.; Lu, M.-X.; Du, Y.-Z. No evidence for an effect of Wolbachia on mtDNA variation and evolution in natural populations of Sesamia inferens (Lepidoptera: Noctuidae). J. Integr. Agric. 2019, 18, 1050-1063. [CrossRef]

33. Nachappa, P.; Levy, J.; Tamborindeguy, C. Transcriptome analyses of Bactericera cockerelli adults in response to "Candidatus Liberibacter solanacearum" infection. Mol. Genet. Genom. 2012, 287, 803-817. [CrossRef] [PubMed]

34. Simonet, P.; Gaget, K.; Balmand, S.; Lopes, M.R.; Parisot, N.; Buhler, K.; Duport, G.; Vulsteke, V.; Febvay, G.; Heddi, A. Bacteriocyte cell death in the pea aphid/Buchnera symbiotic system. Proc. Natl. Acad. Sci. USA 2018, 115, E1819-E1828. [CrossRef]

35. Gerardo, N.M.; Altincicek, B.; Anselme, C.; Atamian, H.; Barribeau, S.M.; De Vos, M.; Duncan, E.J.; Evans, J.D.; Gabaldón, T.; Ghanim, M. Immunity and other defenses in pea aphids, Acyrthosiphon pisum. Genome Biol. 2010, 11, R21. [CrossRef]

36. Chen, W.; Hasegawa, D.K.; Kaur, N.; Kliot, A.; Pinheiro, P.V.; Luan, J.; Stensmyr, M.C.; Zheng, Y.; Liu, W.; Sun, H. The draft genome of whitefly Bemisia tabaci MEAM1, a global crop pest, provides novel insights into virus transmission, host adaptation, and insecticide resistance. BMC Biol. 2016, 14, 110. [CrossRef]

37. Xie, W.; Chen, C.; Yang, Z.; Guo, L.; Yang, X.; Wang, D.; Chen, M.; Huang, J.; Wen, Y.; Zeng, Y. Genome sequencing of the sweetpotato whitefly Bemisia tabaci MED/Q. GigaScience 2017, 6, gix018. [CrossRef] [PubMed]

38. Hunter, W.B.; Reese, J. International Psyllid Genome Consortium. The Asian citrus psyllid genome (Diaphorina citri, Hemiptera). J. Citrus Pathol. 2014, 1, 1. [CrossRef]

39. Yao, J.; Saenkham, P.; Levy, J.; Ibanez, F.; Noroy, C.; Mendoza, A.; Huot, O.; MEYER, D.F.; Tamborindeguy, C. Interactions 'Candidatus Liberibacter solanacearum'-Bactericera cockerelli: Haplotype effect on vector fitness and gene expression analyses. Front. Cell. Infect. Microbiol. 2016, 6, 62. [CrossRef] [PubMed]

40. Nachappa, P.; Levy, J.; Pierson, E.; Tamborindeguy, C. Correlation between "Candidatus Liberibacter solanacearum" infection levels and fecundity in its psyllid vector. J. Invertebr. Pathol. 2014, 115, 55-61. [CrossRef]

41. Li, W.; Abad, J.A.; French-Monar, R.D.; Rascoe, J.; Wen, A.; Gudmestad, N.C.; Secor, G.A.; Lee, M.; Duan, Y.; Levy, L. Multiplex real-time PCR for detection, identification and quantification of 'Candidatus Liberibacter solanacearum'in potato plants with zebra chip. J. Microbiol. Methods 2009, 78, 59-65. [CrossRef] [PubMed]

42. Érdi, B.; Nagy, P.; Zvara, Á.; Varga, Á.; Pircs, K.; Ménesi, D.; Puskás, L.G.; Juhász, G. Loss of the starvation-induced gene Rack1 leads to glycogen deficiency and impaired autophagic responses in Drosophila. Autophagy 2012, 8, 1124-1135. [CrossRef]

43. Ibanez, F.; Hancock, J.; Tamborindeguy, C. Identification and expression analysis of aquaporins in the potato psyllid, Bactericera cockerelli. PLoS ONE 2014, 9, e111745. [CrossRef] [PubMed]

44. Schmittgen, T.D.; Livak, K.J. Analyzing real-time PCR data by the comparative CT method. Nat. Protoc. 2008, 3, 1101-1108. [CrossRef]

45. Ibanez, F.; Tamborindeguy, C. Selection of reference genes for expression analysis in the potato psyllid, Bactericera cockerelli. Insect Mol. Biol. 2016, 25, 227-238. [CrossRef]

46. Levine, B.; Mizushima, N.; Virgin, H.W. Autophagy in immunity and inflammation. Nature 2011, 469, 323. [CrossRef] [PubMed]

47. Mitchell, G.; Cheng, M.I.; Chen, C.; Nguyen, B.N.; Whiteley, A.T.; Kianian, S.; Cox, J.S.; Green, D.R.; McDonald, K.L.; Portnoy, D.A. Listeria monocytogenes triggers noncanonical autophagy upon phagocytosis, but avoids subsequent growth-restricting xenophagy. Proc. Natl. Acad. Sci. USA 2018, 115, E210-E217. [CrossRef] [PubMed]

48. Krokowski, S.; Mostowy, S. Interactions between Shigella flexneri and the autophagy machinery. Front. Cell. Infect. Microbiol. 2016, 6, 17. [CrossRef]

49. Sorbara, M.T.; Ellison, L.K.; Ramjeet, M.; Travassos, L.H.; Jones, N.L.; Girardin, S.E.; Philpott, D.J. The protein ATG16L1 suppresses inflammatory cytokines induced by the intracellular sensors Nod1 and Nod2 in an autophagy-independent manner. Immunity 2013, 39, 858-873. [CrossRef]

50. Ren, C.; Finkel, S.E.; Tower, J. Conditional inhibition of autophagy genes in adult Drosophila impairs immunity without compromising longevity. Exp. Gerontol. 2009, 44, 228-235. [CrossRef]

51. Miller, C.; Celli, J. Avoidance and subversion of eukaryotic homeostatic autophagy mechanisms by bacterial pathogens. J. Mol. Biol. 2016, 428, 3387-3398. [CrossRef] 
52. Kuo, C.-J.; Hansen, M.; Troemel, E. Autophagy and innate immunity: Insights from invertebrate model organisms. Autophagy 2018, 14, 233-242. [CrossRef] [PubMed]

53. Gelino, S.; Hansen, M. Autophagy-an emerging anti-aging mechanism. J. Clin. Exp. Pathol. 2012, Suppl. 4, 006. [CrossRef]

54. Huang, J.; Brumell, J.H. Bacteria-autophagy interplay: A battle for survival. Nat. Rev. Microbiol. 2014, 12, 101. [CrossRef] [PubMed]

55. Biering, S.B.; Choi, J.; Halstrom, R.A.; Brown, H.M.; Beatty, W.L.; Lee, S.; McCune, B.T.; Dominici, E.; Williams, L.E.; Orchard, R.C. Viral replication complexes are targeted by LC3-guided interferon-inducible GTPases. Cell Host Microbe 2017, 22, 74-85.e77. [CrossRef] [PubMed]

56. Kageyama, S.; Omori, H.; Saitoh, T.; Sone, T.; Guan, J.-L.; Akira, S.; Imamoto, F.; Noda, T.; Yoshimori, T. The LC3 recruitment mechanism is separate from Atg9L1-dependent membrane formation in the autophagic response against Salmonella. Mol. Biol. Cell 2011, 22, 2290-2300. [CrossRef] [PubMed]

57. Bernard, A.; Jin, M.; Xu, Z.; Klionsky, D.J. A large-scale analysis of autophagy-related gene expression identifies new regulators of autophagy. Autophagy 2015, 11, 2114-2122. [CrossRef]

58. Choy, A.; Dancourt, J.; Mugo, B.; O'Connor, T.J.; Isberg, R.R.; Melia, T.J.; Roy, C.R. The Legionella effector RavZ inhibits host autophagy through irreversible Atg8 deconjugation. Science 2012, 338, 1072-1076. [CrossRef] [PubMed]

59. Mendoza Herrera, A.; Levy, J.; Harrison, K.; Yao, J.; Ibanez, F.; Tamborindeguy, C. Infection by Candidatus Liberibacter solanacearum'haplotypes A and B in Solanum lycopersicum'Moneymaker'. Plant. Dis. 2018, 102, 2009-2015. [CrossRef]

60. Harrison, K.; Tamborindeguy, C.; Scheuring, D.C.; Herrera, A.M.; Silva, A.; Badillo-Vargas, I.E.; Miller, J.C.; Levy, J.G. Differences in Zebra Chip Severity between 'Candidatus Liberibacter Solanacearum'Haplotypes in Texas. Am. J. Potato Res. 2019, 96, 86-93. [CrossRef] 